\title{
Generalized sub band analysis and signal synthesis
}

\author{
Evgeny G. Zhilyakov, Sergei P. Belov, Ivan I. Oleinik, Sergei L. Babarinov, Diana I. Trubits yna \\ Belgorod State National Research University, Russia
}

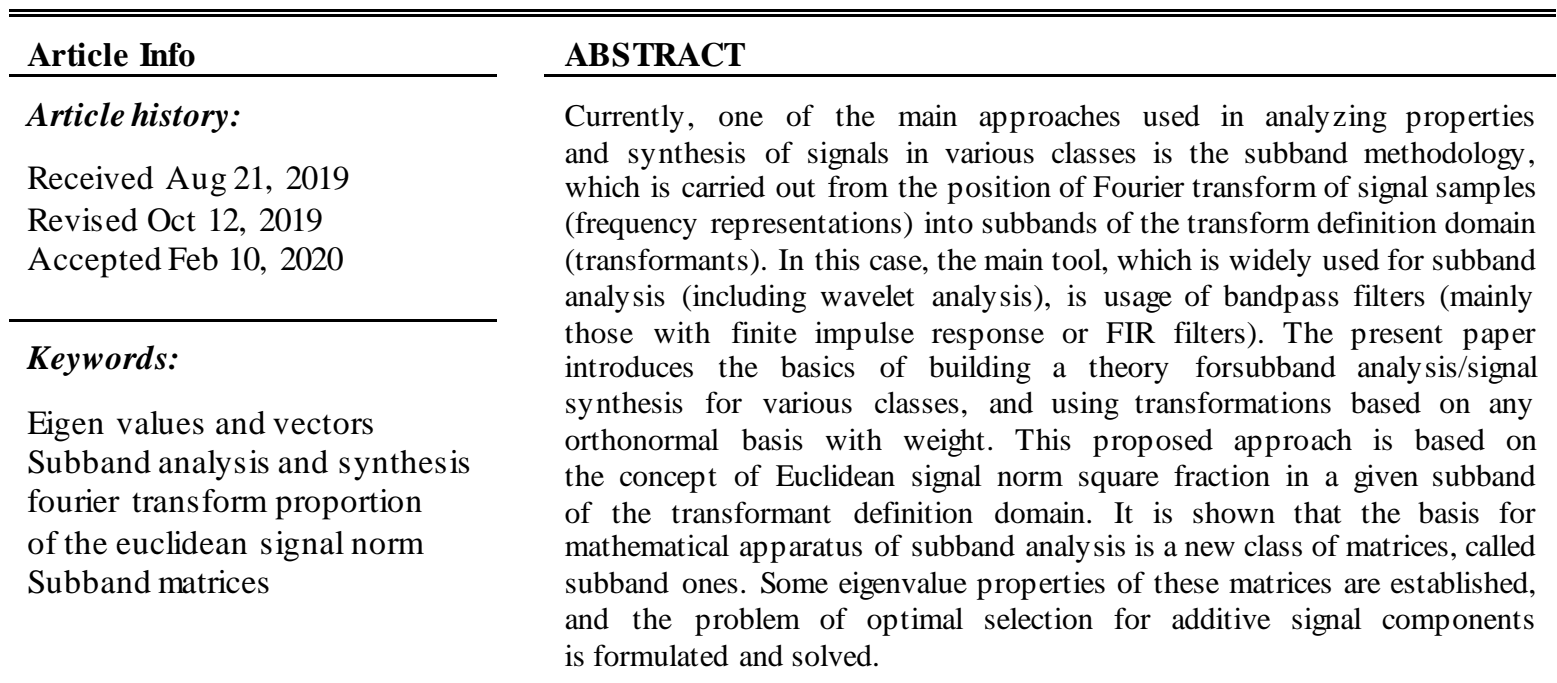

This is an open access article under the $\underline{C C B Y-S A}$ license.

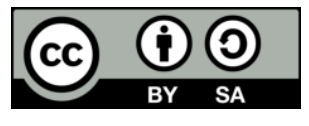

\section{Corresponding Author:}

Evgeny G. Zhilyakov,

Belgorod State National Research University,

85 Pobedy Street, Belgorod, the Belgorod region, 308015, Russia.

Email: zhilyakov@bsu.edu.ru

\section{INTRODUCTION}

As an important object of analysis and synthesis, you can also specify speech signals that are recorded at the outputs of microphones under the acoustic influence of oral speech [1-7]. Their distinguishing feature is the non-stationarity due to alternation of speech sounds. Therefore, the processing of finite dimension vectors adequately reflects this property.

The degree of concentration of their Euclidean norms (energies) is often used as the most important criterion in synthesis of signals, in some subdomain of the used transformation definition, for example, Fourier [8]. Thus, description of the signals properties from standpoint of splitting their transformations definitions domains into subregions, is a useful technique for analysis and synthesis of them. Such an analysis and synthesis seemto be called subband.

Subband analysis and synthesis is widely used in framework of the Fourier transform. However, one cannot assume that it is optimized from the standpoint of estimating the accuracy of the signals subband characteristics. In particular, the existing theoretical foundations of subband analysis/synthesis are not sufficiently developed, since only digital filtering issues are sufficiently developed. However, even in this case, aspects of informativity are not sufficiently developed from the standpoint of distortions, which affect interpretation of the results obtained of the used process ing, for example, accuracy of Euclidean signal rate fraction determination in a given subregion (subband) of the Fourier transform definition domain. Thus, development of subband analysis/synthesis methods from the standpoint of achieving high information content from the obtained results is relevant. 
It is also obvious that the use of Fourier basis, although important, is not the only way to represent signals in the corresponding spaces, which make it possible to display the properties of signals that do not appear clearly in the time domain. Therefore, it seems appropriate to develop fundamentals of the subband analysis and synthesis theory for a certain class of transformations based on orthogonal bases. The solution of this problem is the content of the present work. It has been shown that the subband properties of signals are adequately described using the subband matrix apparatus, whose eigenvectors are a natural orthonormal basis in the space of real vectors of the corresponding dimension.

\section{METHODOLOGY}

In this work, it is assumed that the vector $\vec{x}=\left(x_{1}, . ., x_{N}\right)^{\prime}$, where the prime means transposition, consists of components obtained either as a result of an equidistant discretization of a continuous signal (a function of time).

$$
x(t), t=[0, T], T=(N-1) \Delta t
$$

In steps $\Delta t$, or from components obtained as a result of synthesis on the basis of a certain criterion, for example, during the formation of signal-code constructions in the information transmission systems. This refers to compliance, and the boundedness condition of the Euclidean norms.

$$
\begin{aligned}
& x_{k}=x(k \Delta t), k=1, . ., N \\
& \|\vec{x}\|=\left(\sum_{k=1}^{N} x_{k}^{2}\right)^{1 / 2}<\infty
\end{aligned}
$$

The widest used methods of analysis can be attributed to separation of the signal into additive components.

$$
\vec{x}=\sum_{r=1}^{R} \vec{y}_{r}
$$

Which meet the specified requirements and selection (filtering) of these components. Based on this technique, the tasks of extracting components from information signals during multichannel information processing [1-3], as well as cleaning signals from the distorting effects of random noise [4, 5], are solved. Moreover, note that one of the most important tasks of analyzing empirical data is detection and recognition of signals, for which the selection of information components is used as well [6].

\section{RESULTS}

Fundamentations of the generalized subband analysis and synthesis theorfor symbol F means the set in the general case of complex functions,

$$
F=\left\{\phi_{n}(t), t \in(a, b) ; n=0,1, \ldots\right\}
$$

that satisfy orthonormal conditions,

$$
s_{n m}=\left(\phi_{n}, \phi_{m}\right)=\int_{a}^{b} \phi_{n}(t) \phi_{b}^{*}(t) w(t) d t=\delta_{n m},
$$

where the asterisk denotes complex conjugation; $\delta_{n m}$ is $\operatorname{Kronecker} \operatorname{symbol} ;(a, b)$ is scope, including, possibly, the whole numerical axis; $w(t)$ - non-negative weight function

$$
w(t) \geq 0, t \in(a, b)
$$


For simplicity, such functions will be called basic. Examples of such bases commonly used in signal processing are the followings:

Exponential Functions

$$
\phi_{n}(t)=\exp (-j t n), t \in(-\pi, \pi) ; n=0, \ldots, w(t) \equiv 1
$$

Cosine basis

$$
\phi_{0}(t)=1 / \pi ; \phi_{n}(\mathrm{t})=2 \cos (t n) / \pi, t \in(-\pi, \pi) ; n=1, \ldots, w(t) \equiv 1
$$

Sine base

$$
\phi_{0}(t)=0 ; \phi_{n}(\mathrm{t})=2 \sin (t n) / \pi, t \in(-\pi, \pi) ; n=1, \ldots, w(t) \equiv 1
$$

Chebyshevpolynomials the first kind $[8,9]$

$$
\phi_{0}(t)=1 / \pi ; \phi_{n}(\mathrm{t})=2 \cos (n a \cos (t)) / \pi, t \in(-1,1) ; n=1, \ldots, w(t)=\left(1-t^{2}\right)^{-1 / 2}
$$

Hermite Polynomials [9]

$$
\begin{aligned}
& \phi_{n}(t)=(-1)^{\mathrm{n}} \exp \left(t^{2} / 2\right) d \exp \left(-t^{2} / 2\right) / d t, t \in(-\infty, \infty) ; n=0, \ldots \\
& w(t)=\exp \left(-t^{2} / 2\right)
\end{aligned}
$$

Bessel functions [9]

$$
\phi_{n}(t)=J_{v}\left(\mu_{n} t / b\right), t \in(0, b) ; n=1, . . ; w(t)=t /\left(b^{2} J_{v+1}^{2}\left(\mu_{n} .\right)\right) ; v \geq 0
$$

where $\mu_{\mathrm{n}}, n=1, \ldots$ - zeros of the Bessel function the first kind $J_{v}, \mathrm{v} \geq 0$. In the framework of this work, the linear state of the form is called as the transformation of source data (vector (2))

$$
X(t)=\sum_{n=1}^{N} x_{n} \phi_{n}(t)
$$

In accordance with the established terminology, the left part of (14) can be called transformant of the original data in the chosen basis or simply the transformant. Based on the property (6), the representation is valid (inversetransformation).

$$
x_{k}=\int_{a}^{b} X(t) \phi_{k}^{*}(t) w(t) d t
$$

It is also obvious that, based on the property (6), the following equality is held,

$$
\|\vec{x}\|^{2}=\sum_{k=1}^{N} x_{k}^{2}=\int_{a}^{b} w(t)|X(t)|^{2} d t,
$$

Which is a generalization of the Parseval equality for Fourier transform [10]. It can be represented in the following sub-band form. 


$$
\|\vec{x}\|^{2}=\sum_{r=1}^{R} P_{r}(\vec{x})
$$

Where terms are determined by the following integrals

$$
P_{r}(\vec{x})=\int_{t \in T_{r}} w(t)|X(t)|^{2} d t
$$

For real bases by intervals (subbands) of the form

$$
T_{r}=\left(a_{r}, b_{r}\right), a \leq a_{r}<b_{r} \leq b ; r=1, \ldots, R
$$

or symmetric unions for complex bases

$$
T_{r}=\left(-b_{r},-a_{r}\right) \bigcup\left(a_{r}, b_{r}\right)
$$

It is assumed that the number of such subbands is finite, although their width may be unlimited, for example, in the case of using Hermite polynomials. It is obvious that (18) thform integrals determine the distribution of Euclidean norms (energies) squares, obtained of the empirical data and can serve as an important tool for their analysis. It is not difficult to get their representation directly in domain of the source data. To do this, substitute the representation (14) in the definition (18). As a result of simple transformations, we obtain the following quadratic form,

$$
P_{r}(\vec{x})=\bar{x}^{\prime} C_{r} \vec{x},
$$

where $C_{r}=\left\{c_{i k}^{r}\right], i, k=1, . ., N$ - symmetric matrix with real elements

$$
c_{i k}^{r}=\int_{t \in T_{r}} w(t) \phi_{i}(t) \phi_{k}^{*}(t) d t
$$

It is also clear that, since characteristics (18) are not negative, the quadratic forms (20) are non-negative, that is, matrices with elements (21) are non-negatively defined. Therefore [11] they can be represented as

$$
C_{\mathrm{r}}=Q_{r} L_{r} Q_{r}^{\prime}
$$

where $Q_{r}$ is orthogonal matrix of real eigenvectors

$$
Q_{r}^{\prime} Q_{r}=Q_{r} Q_{r}^{\prime}=I=\operatorname{diag}(1, \ldots, 1),
$$

which is a solution to (24)

$$
C_{r} Q_{r}=Q_{r} L_{r}
$$

But $L_{r}=\operatorname{diag}\left(\lambda_{1}, \ldots, \lambda_{N}\right)$ - diagonal matrix of real eigenvalues, which we assume ordered in descending order

$$
\lambda_{k}^{r} \geq \lambda_{k+1}^{r} \geq 0, k=1, \ldots, N-1
$$

In the following, matrices with elements of the form (21) are called subband ones. In addition to relations (22), (23) and (25), some more specific relations for their own numbers and vectors can also be 
obtained. In accordance to the definition (24), equality must be maintained for the individual components of the eigenvectors $\vec{q}_{k}^{r}=\left(q_{1 k}^{r}, \ldots, q_{N k}^{r}\right)^{\prime}$

$$
\begin{aligned}
& \lambda_{k}^{r} q_{i k}^{r}=\sum_{m=1}^{N} c_{i m}^{r} q_{m k}^{r}, \\
& \lambda_{k}^{r} q_{i k}^{r}=\int_{t \in T_{r}} w(t) \phi_{i}(t) G_{k}^{r^{*}}(t) d t
\end{aligned}
$$

Substitution into which representations (21) gives, where the function under the integral is a complex conjugate transformant of an eigenvector

$$
G_{k}^{r}(t)=\sum_{m=1}^{N} \phi_{m}(t) q_{m k}^{r}
$$

Relation (25) allows to get an idea for projection on the eigenvector (scalar product) of the initial vector

$$
\lambda_{k}^{r} d_{k}^{r}(\vec{x})=\lambda_{k}^{r}\left(\vec{x}, \vec{q}_{k}^{r}\right)=\lambda_{k}^{r} \sum_{m=1}^{N} x_{i} q_{i k}^{r}=\int_{t \in T_{r}} w(t) X(t) G_{k}^{r^{*}}(t) d t
$$

Note that projection is determined by transformant segments of the eigen and original vectors in the selected subband. Similarly, you can get equality

$$
\lambda_{k}^{r} d_{k}^{r}\left(\vec{q}_{n}\right)=\lambda_{k}^{r}\left(\vec{q}_{n}^{r}, \vec{q}_{k}^{r}\right)=\int_{t \in T_{r}} w(t) G_{n}^{r}(t) G_{k}^{r^{*}}(t) d t
$$

From here, taking into account the orthonormal eigenvectors (21), we obtain that for the segments of the transformants of eigenvectors the following equalities are held. Transformants of various eigenvectors of

$$
n \neq k
$$

have the property of double orthogonality [10] (simultaneous orthogonality throughout the domain and its subband),

$$
\int_{a}^{b} w(t) G_{n}^{r}(t) G_{k}^{r^{*}}(t) d t=\int_{t \in T_{r}} w(t) G_{n}^{r}(t) G_{k}^{r^{*}}(t) d t=0,
$$

While the eigenvalue determines fraction of square of the norm of the corresponding eigenvector falling into the subband

$$
\lambda_{k}^{r}=\int_{t \in T_{r}} w(t)\left|G_{n}^{r}(t)\right|^{2} d t \leq \int_{a}^{b} w(t)\left|G_{n}^{r}\right|^{2} d t=1, n=1, \ldots, N
$$

Note that the equality to the unit of the second integral is obtained on the basis of the Parseval equality (17) and the orthonormal nature of the eigenvectors. Bearing in mind the decomposition (22) and definition (29), from (20) it is not difficult to get an idea for the characteristic (18)

$$
P_{r}(\vec{x})=\sum_{k=1}^{N} \lambda_{k}^{r} d_{k}^{r 2}(\vec{x})
$$


which allows us to simplify and in particular, parallelize the procedure for calculating quadratic forms, when the eigenvectors are pre-computed for their repeated use. When dividing the original vector into additive components (see (4)), you can use the ideal requirement

$$
Y_{r}(t)=X(t), t \in T_{r} ; Y_{r}(t) \equiv 0, t \notin T_{r},
$$

where $Y_{r}(t)$ - transformant vector

$$
\vec{y}_{r}=\left(y_{1 r}, \ldots, y_{N r}\right)^{\prime} .
$$

Obviously, the requirement (35) can be fulfilled exactly if the subband is equal to the entire domain of the transformant. Therefore, it seems appropriate to use the following functionality as a measure for the error in its implementation.

$$
E\left(\vec{x}, \vec{y}_{r}\right)=\beta \int_{t \in T_{r}} w(t)\left|X(t)-Y_{r}(t)\right|^{2} d t+(1-\beta) \int_{t \notin T_{r}}\left|Y_{r}(t)\right|^{2} d t
$$

where the parameter

$$
0<\beta<1
$$

Serves to set the weight (importance) of the components. You can show the view vector

$$
\vec{y}_{r}=\beta\left((1-\beta) I+(2 \beta-1) C_{r}\right)^{-1} C_{r} \vec{x} .
$$

In case of equal weights,

$$
\beta=0,5,
$$

relation (39) gives

$$
\vec{y}_{r}=C_{r} \vec{x}
$$

From here, taking into account (21), we obtain the following representation for the components of the resulting vector;

$$
y_{k r}=\int_{t \in T_{r}} w(t) \phi_{k}^{*}(t) X(t) d t
$$

Thus, the components of the resulting vector are completely determined by the segment of the transformant of the original data in the selected subband. Obviously, this conclusion can be extended to the vector (39), since it is completely determined by the vector (41). Based on the definition of (21), we obtain the equality

$$
\sum_{r=1}^{R} C_{r}=I
$$

when subbands are adjacent to each other and cover the entire domain. Obviously, this corresponds to obtaining components of the vector that will be analyzed, which meet the requirement of components additivity (4). The problem of signal synthesis with specified properties is often considered during transmission of information and remote control. The criterion of energy concentration (the square of the Euclidean norm) is widely used in a certain subband of the entire frequency band. This criterion is easy to generalize in the case of any transformations, and to formulate the problem of finding a vector that maximizes the characteristic (20)

$$
\overline{\mathrm{Z}} C_{r} \vec{z}=\max \vec{x} C_{r} \vec{x}, \forall \vec{x} \in R^{N}
$$


The solution of this problem is the matrix corresponding eigenvector with elements (21) corresponding for the maximu $\mathrm{m}$ eigenvalue.

$$
\mathrm{Z}=\bar{q}_{1}^{r}
$$

It is clear that subband matrices serve as the basis for solving the considered problems of generalized subband analysis / synthesis of vectors. In the case of symmetry and non-negative definiteness, they have a complete set of eigenvectors that can serve as a basis of space. These bases are determined by the type of the transformant and the selected subband of its domain. Therefore, it is natural to call them subband. It is interesting to consider specific examples of subband matrices and their eigenvalues and vectors. For some types of transformations, it is possible to obtain analytical relations for the elements of subband matrices.

a. Exponential basis (8).

The elements of the subband matrix (21) in this case can be represented in an analytical form.

$$
c_{i k}^{r}=2 \sin \left(\Delta_{r}(i-k)\right) \cos \left(\omega_{r}(i-k)\right) / \pi(i-k),
$$

where

$$
\Delta_{r}=\left(b_{r}-a_{r}\right) / 2 ; \omega_{r}=\left(b_{r}+a_{r}\right) / 2 .
$$

The main properties of subband matrices and their eigenvectors were considered in [12]. It also discusses the features of using this apparatus for solving some problems of analysis and signal synthesis. In [13], it was shown that the subband methodology can be successfully applied under conditions of complete, in which there is a priori uncertainty in identifying trends of empirical data based on adaptive filtering. It does not use a priori postulation for the analytical form of the functional dependence of the trend. b. Cosine basis (9)

The elements of the subband matrix are represented as

$$
c_{i k}^{r}=2 \sin \left(\Delta_{r}(i-k)\right) \cos \left(\omega_{r}(i-k)\right) / \pi(i-k)+2 \sin \left(\Delta_{r}(i+k)\right) \cos \left(\omega_{r}(i+k)\right) / \pi(i+k)
$$

Relation (21), after substituting the definition (11) into it and replacing the variable $t=\cos (z)$ gives a ratio similar to (48), with the difference that instead of (47), the parameters are determined from the relations

$$
\Delta_{r}=\left(a \cos \left(b_{r}\right)-a \cos \left(a_{r}\right)\right) / 2 ; \omega_{r}=\left(a \cos \left(b_{r}\right)+a \cos \left(a_{r}\right)\right) / 2
$$

\section{DISCUSSION}

Current methodologyfor subband analysis of empirical data and synthesis of signals with desired properties is developed regarding the problems needed to be solved from the standpoint of subdividing domain of the Fourier transform based on the exponential basis (8) (frequency representations). Frequency representations also built developing methods of wavelet analysis quite intensively, for which they use filters with finite impulse response, calculated in a quite specific way [14]. This is in favor of the conclusion that the sub-band methodology is adequate for the tasks of signal $\mathrm{V}$ analysis and synthesis.

At the same time, for some empirical data and signals, the description using other bases (for example Bessel functions) may be adequate when analyzing oscillations of circular membranes $[15,16]$. Therefore, it is important to develop the mathematical foundations ofsubband analysis and signal synthesis from the standpoint of partitioning transformation regions into subbands of other transformations using orthonormal bases. This allows for a completely regular way to implement the subband methodology in these cases, as well [17-25].

\section{CONCLUSION}

In this paper, the theoretical foundations of subband analysis are developed from the standpoint of dividing the transformation definitions domains (transformants) of the initial sampled data into subbands, according to orthonormal bases. It is shown that special matrices, which are naturally called subband ones, 
are the main elements of subband analysis. It is also indicated that subband matrices are symmetric and positive definite. Thus, the set of their eigenvectors is a complete orthonormal basis. It is natural to use it as the main tool forsubband analysis / synthesis. A criterion is formulated that defines the optimal subband filtering of signals on additive components, and a solution is obtained for the corresponding variat ional problem. It is shown that the selected components have an important property, and they are completely determined by segment of the transformant in a given subband. The conditions that allow to restore the original signal based on a simple summation of the selected components are revealed. Besides, the relations defining the elements of subband matrices for some widely used bases are presented.

\section{ACKNOWLEDGMENT}

The present work was done with financial support from the Ministry of Education and Science, in the state assignment framework of Belarusian State University (Project \# 8.2201.2017 / 4.6)

\section{REFERENCES}

[1] S. A. Farshad and M. Sheikholeslami, "Simulation of exergy loss of nanomaterial through a solar heat exchanger with insertion of multi-channel twisted tape," Journal of Thermal Analysis and Calorimetry, vol. 138, no. 1, pp. 75-804, 2019.

[2] R. M. Blakar, "Towards a theory of communication in terms of preconditions: A conceptual framework and some empirical explorations," in Recent advances in language, communication, and social psychology, pp. 10-40, 2018.

[3] V. Oreshkov, et al., "Estimation of the guard interval duration variation effectiveness in the orthogonal harmonic signals transmission systems," in 2017 14th International Conference The Experience of Designing and Application of CAD Systems in Microelectronics (CADSM), pp. 109-112, 2017.

[4] G. E. Gorbet, et al., "Multi-speed sedimentation velocity implementation in UltraScan-III," European Biophysics Journal, vol. 47, no.7, pp. 825-835, 2018.

[5] E. G. Zhilyakov, et al., "Subband analysis and synthesis of signals," Compusoft, An International Journal of Advanced Computer Technology, vol. 8, no. 6, pp. 3206-3211, 2019.

[6] V. Golikov, et al., "Robust multipixel matched subspace detection with signal-dependent background power," Journal of Applied Remote Sensing, vol. 10, no. 1, pp. 015006, 2016.

[7] T. N. Balabanova, et al., "The using of orthogonal basis for the steganographic coding of information in multimedia," Research Result: Information Technologies, vol. 2, no. 2, pp. 40-47, 2017.

[8] T. M. Chan, "Applications of Chebyshev polynomials to low-dimensional computational geometry," in 33rd International Symposium on Computational Geometry (SoCG 2017), 2017.

[9] A. I. Zayed, "Handbook of function and generalized function transformations," CRC press, 2019.

[10] Yakovlev V. P. and Khurgin Y. I., "Finite functions in physics and technology," Knizhnyy dom LIBROKOM, 2010.

[11] J. Bun, et al., "Cleaning large correlation matrices: tools from random matrix theory," Physics Reports, vol. 666, pp. 1-109, 2017.

[12] E. G. Zhily akov, "Optimal sub-band methods for analy sis and synthesis of finite-duration signals," Automation and remote control, vol. 76, no. 4, pp. 589-602, 2015.

[13] Y. Song, et al., "Trends and opportunities of BIM-GIS integration in the architecture, engineering and construction industry: a review from a spatio-temporal statistical perspective," ISPRS International Journal of Geo-Information, vol. 6 , no. 12, p. 397-428, 2017.

[14] S. Mallat, "A Wavelet Tour of Signal Processing," 3rd Edition, New York, Academic Press, 2009.

[15] N. Jalalinezhad and H. Jenaabadi, "Studying Effect of Communication Skills and Leadership Styles of Manager on Knowledge Management of Zahedan University of Medical Sciences, Iran," Journal of Management and Accounting Studies, vol. 2, no. 2, pp.31-37, 2014.

[16] A. Jalili, et al., "Firefly algorithm based on fuzzy mechanism for optimal congestion management," Journal of Research in Science, Engineering and Technology, vol. 3, no. 3, pp. 1-7, 2015.

[17] S. Surender, et al., "6-bit, 180nm Digital to Analog Converter (DAC) Using Tanner EDA Tool for Low Power Applications," International Journal of Communication and Computer Technologies, vol. 5, no. 2, pp. 82-88, 2017.

[18] N. M. Rao, et al., "RP-HPLC method development and validation for estimation of Voglibose in bulk and tablet dosage forms," International Journal of Research in Pharmaceutical Sciences, vol. 1, no. 2, pp. 190-194, 2010.

[19] P. Peres, et al., "Are Really Technologies at the Fingers of Teachers? Results from a Higher Education Institution in Portugal," Journal of Information Systems Engineering \& Management, vol. 3, no. 1, pp. 08-16, 2018.

[20] V. Madanipour, et al., "Multi-objective component sizing of plug-in hybrid electric vehicle for optimal energy management," Clean Technologies and Environmental Policy, vol. 18, no. 4, pp. 1189-202, 2016. 
[21] Y. Feng, et al., “A Novel Monarch Butterfly Optimization with Global Position Updating Operator for Large-Scale 0-1 Knapsack Problems," Mathematics, vol. 7, no. 11, pp. 1056-1086, 2019.

[22] J. H. Yap and Y. C. Wong, "A 30mV input battery-less power management system," Bulletin of Electrical Engineering and Informatics, vol. 8, no. 4, pp. 1169-1179, 2019.

[23] H. Wang, et al., "The impact of HVDC links on transmission system collapse," in 2017 IEEE Conference on Energy Internet and Energy System Integration (EI2), pp. 1-6, 2017.

[24] G. Sinha, et al., “A Comparative Strategy Using PI \& Fuzzy Controller for Optimization of Power Quality Control,” Indonesian Journal of Electrical Engineering and Informatics (IJEEI), vol. 6, no. 1, pp. 118-124, 2018.

[25] M. Montazeri G. and K. M. Mahmoodi, "Optimized predictive energy management of plug-in hybrid electric vehicle based on traffic condition," Journal of cleaner production, vol. 139, pp.935-948, 2016.

\section{BIOGRAPHIES OF AUTHORS}
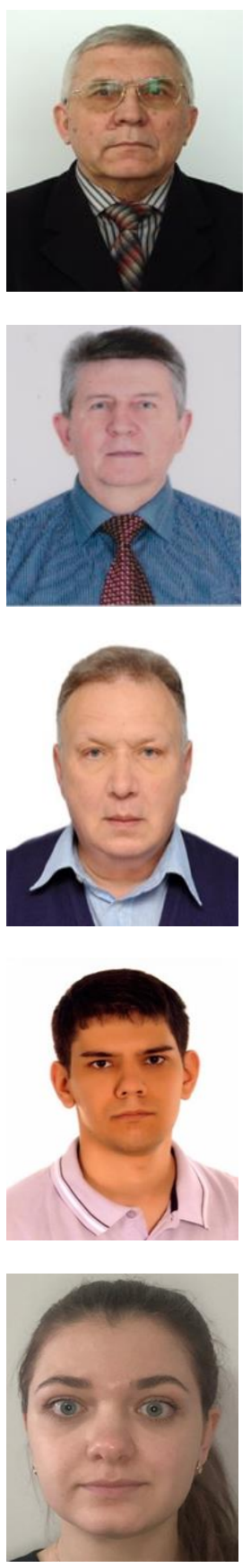

Evgeny G. Zhilyakov received the degree of Doctor of Technical Sciences in 1994. In 1997 he was awarded the academic title of professor. He is currently head of the Department of Belgorod State National Research University. His research interests include signal analy sis and synthesis theory, methods for minimizing the costs of time-frequency resources of information transmission channels

Sergei P. Belov received a doctorate in technical sciences in 2012. In 2015, he was awarded the academic title of professor. Currently he is a professor at the Department of Information and Telecommunication Systems and Technologies, Belgorod State National Research University. His research interests include the theory of the formation and processing of channel signals, methods of minimizing the costs of time-frequency resources of information transmission channels

Ivan I. Oleinik received the degree of Candidate of Technical Sciences in 2003. He is currently working on his thesis. $\mathrm{He}$ is a senior lecturer at the Department of Information and Telecommunication Systems and Technologies, Belgorod State National Research University. His research interests include the processing of radar information, the development of decision rules for the recognition of objects of radar observation

Sergei L. Babarinov received a degree in communications networks and switching systems at the Belgorod State National Research University in 2011. In 2018, he received the degree of candidate of technical sciences. He is a senior lecturer at the Department of Information and Telecommunication Systems and Technologies. His research interests include signal analy sis and synthesis methods.

Diana I. Trubitsyna received a bachelor's degree in engineering and technology in 2013, and in 2015 received a master's degree in information and communication technologies and communication systems at the Belgorod State National Research University. Currently, she is preparing to defend a Ph.D. thesis on the topic of subband compression of speech signals in the field of determining the cosine transform. Her research interests include signal analysis and synthesis methods. 